\title{
Heavy-ion collisions in a fixed target mode at the LHC beams
}

\author{
Alexey Kurepin ${ }^{1, \star}$ and Nataliya Topilskaya ${ }^{1, \star \star}$ \\ ${ }^{1}$ Institute for Nuclear Research, RAS, Moscow, Russia
}

\begin{abstract}
The interaction of high-energy LHC beams with fixed target, including polarized nuclei targets, can expand the range of fundamental physical investigations accessible at CERN. High-intensity beam of protons and lead ions accumulated in the LHC collider allows to apply the gas-target system like the LHCb SMOG or HERMES systems. It is also possible to use the beam halo by placing in the halo the fixed target in the form of thin ribbon or use a bend crystal to extract the beam. In the extracted beam it is possible to install a polarized target. Using the proton and ion beams of the LHC with fixed targets, the data in the energy interval between maximum energy of the SPS and the nominal RHIC energy in p-A and A-A collisions could be obtained. The fixed target mode allows the intensive study of rare processes, the study of polarization phenomena, the measurements of the parameters needed to analyze the data of cosmic rays and neutrino astrophysics, detailed study of the processes of quarkonia production and suppression. The high statistics data on quarkonium production at these energies will give the possibility to clarify the mechanism of production, to investigate the importance of recombination process and the energy dependence on the phase transition of nuclear matter to the quark-gluon phase. Also the physical program includes the study of the Drell-Yan process, D-meson production, flow and spin physics.
\end{abstract}

\section{Introduction}

At high temperature and at large energy density the existence of Quark-Gluon Plasma (QGP) is predicted by lattice Quantum Chromo Dynamics (QCD). But despite of big success in recent years at RHIC and LHC colliders the properties of nuclear matter at extreme conditions remain poorly understood. Progress in our understanding of the properties of nuclear matter requires systematic and high statistical measurements also in low energy region. Unfortunately, the data produced by the beam energy scan at RHIC have large statistical and systematical errors. The luminosity in collider experiments at RHIC decreases at low energy. The fixed target experiments at the LHC beams will have the number of advantages compared with the experiments on collider. Using the high-intensity LHC beams will significantly increase the statistical accuracy of the data and provide better acceptance and efficiency of measurements. It is possible to study $\mathrm{p}-\mathrm{A}$ and $\mathrm{Pb}-\mathrm{A}$ collisions with different nuclear targets and to use polarized targets. The fixed target experiments give possibility to access to high $x_{\mathrm{F}}$ Feynman domain by measuring the far backward region in the center-of-mass (c.m.) system. If the proton and ion beams would be used at the LHC with the fixed target, the energy interval between SPS

\footnotetext{
$\star$ e-mail: kurepin@inr.ru

$\star \star$ e-mail: topilska@inr.ru
} 
energy $\left(\sqrt{s_{\mathrm{NN}}}=29 \mathrm{GeV}\right)$ and RHIC nominal energy $\left(\sqrt{s_{\mathrm{NN}}}=200 \mathrm{GeV}\right)$ in p-A and A-A collisions could be covered. For proton beam with $450 \mathrm{GeV}$ energy the energy in N-N c.m. is $\sqrt{s_{\mathrm{NN}}}=29.1 \mathrm{GeV}$. For pp and p-A collisions with the $7 \mathrm{TeV}$ proton beam $\sqrt{s_{\mathrm{NN}}}=114.6 \mathrm{GeV}$ with the rapidity shift 4.8 $\left(y_{c . m} .=0 \rightarrow y_{\text {lab }}=4.8\right)$. For $\mathrm{Pb}-\mathrm{p}$ and $\mathrm{Pb}-\mathrm{A}$ collisions using lead beam with energy $2.75 \mathrm{TeV}$ the c.m. N-N energy is $\sqrt{s_{\mathrm{NN}}}=71.8 \mathrm{GeV}$ with the rapidity shift 4.3 . Using LHC beams with reduced energy it would be possible to scan energy range between SPS and RHIC. This is a unique possibility to clarify the mechanism of charmonium, $\mathrm{J} / \psi$ and $\psi(2 \mathrm{~S})$ production, to investigate the contribution of recombination process, since the probability of recombination decreases with decreasing energy of collision, and to search the critical point of the phase transition.

\section{Quarkonium production at the CERN Large Hadron Collider LHC}

At the LHC energies five quarkonium states from two families are under study, charmonia: $\mathrm{J} / \psi$ and $\psi(2 S)$ and bottomonia: $\Upsilon(1 S), \Upsilon(2 S)$ and $\Upsilon(3 S)$. In the color-screening model quarkonium states are suppressed in the QGP with different dissociation probabilities for the various mass states, depending on their binding energy [1]. The CMS Collaboration at the LHC reported the observation of the sequential suppression of bottomonium states in $\mathrm{Pb}-\mathrm{Pb}$ collisions at $\sqrt{s_{\mathrm{NN}}}=2.76 \mathrm{TeV}$ [2,3]. However, other nuclear matter effects besides color screening, as well as cold nuclear matter (CNM) effects, regeneration and feed down from higher states and B-meson decay for $\mathrm{J} / \psi$ production should be taken into account.

The results of recent measurements by the ALICE Collaboration are compatible with a regeneration mechanism playing an important role in the production of $\mathrm{J} / \psi$ in $\mathrm{Pb}-\mathrm{Pb}$ collisions at the $\mathrm{LHC}$ [4-6]. Additional $\mathrm{J} / \psi$ are expected to be produced from deconfined charm quarks by kinetic recombination in the QGP [7] or by statistical hadronization at the phase boundary [8]. Due to the lower production cross section of $b \bar{b}$ pairs compared to $c \bar{c}$ pairs, the regeneration of $\Upsilon(1 \mathrm{~S})$ is expected to be smaller than that of $\mathrm{J} / \psi[9]$.

On the other hand, effects related to the presence of CNM can also modify the production of quarkonia in nucleus-nucleus collisions. Cold nuclear matter effects can be separated into initial and final-state effects. Initial-state effects include shadowing and parton energy loss. The nuclear Parton Distribution Function (nPDF) is different from that of free nucleons (PDF). Since the gluon fusion mechanism dominates in heavy-quarks production, quarkonium production is particularly sensitive to the gluon nPDF, which is presently not well known. The heavy-quark pair can interact with the nuclear matter and eventually break up, that is normal nuclear absorption. The final-state resonance can also interact with surrounding comovers and lose energy or even break up. In four experiments ALICE, ATLAS, CMS and LHCb at the LHC, the quarkonium production was measured in different rapidity and transverse momentum ranges. The $\mathrm{J} / \psi$ production was measured in pp collisions at $\sqrt{s_{\mathrm{NN}}}=2.76$, 7, 8 and $13 \mathrm{TeV}$ and in $\mathrm{Pb}-\mathrm{Pb}$ collisions at $\sqrt{s_{\mathrm{NN}}}=2.76$ and $5.02 \mathrm{TeV}$. The quarkonium production in $\mathrm{p}-\mathrm{Pb}$ collisions at $\sqrt{s_{\mathrm{NN}}}=5.02 \mathrm{TeV}$ was measured for detailed investigation of the cold nuclear matter effects and investigation of production mechanism. In $2016 \mathrm{p}-\mathrm{Pb}$ collisions would be measured also at $\sqrt{s_{\mathrm{NN}}}=8 \mathrm{TeV}$.

The data of the $\mathrm{J} / \psi$ production in pp collision at the same energy as in $\mathrm{Pb}-\mathrm{Pb}$ collision, gives the reference for extracting the nuclear modification factor $R_{A A}$. There is a good agreement for $\mathrm{pp}$ collision between the data obtained in ALICE, LHCb, CMS and ATLAS experiments in the same kinematical domains. The contribution of B-decay to $\mathrm{J} / \psi$ production cross section is approximately $10 \%$ for $p_{\mathrm{T}}$ near $1.5 \mathrm{GeV} / \mathrm{c}$ [11]. It depends on rapidity and increases for larger $\mathrm{J} / \psi$ transverse momentum.

Charmonium production in $\mathrm{Pb}-\mathrm{Pb}$ collisions at 2.76 and 5.02 $\mathrm{TeV}$ was measured in ALICE, CMS and ATLAS experiments. In ALICE experiment the transverse momentum of inclusive $\mathrm{J} / \psi$ mesons 
was measured from values near zero up to $12 \mathrm{GeV} / \mathrm{c}$ in rapidity range $|y|<0.9$ (for $\mathrm{J} / \psi$ decay into two electrons) and $2.5<y<4$ (for muons channel). The comparison of PHENIX and ALICE $R_{A A}$ results is shown in figure $1[6,12]$.
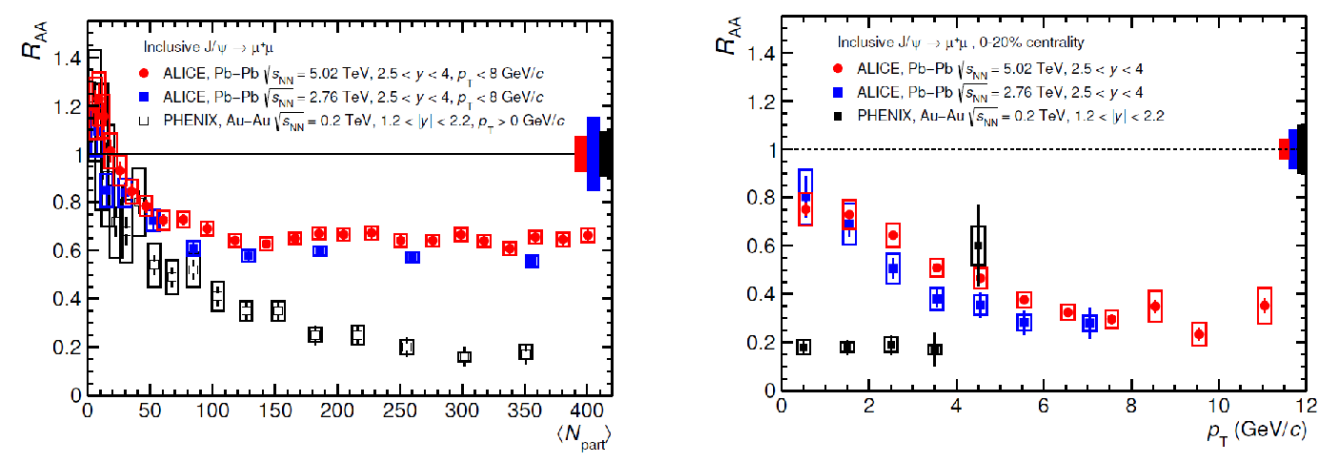

Figure 1. The comparison of PHENIX and ALICE data of $R_{A A}$. The left panel shows $R_{A A}$ depending on centrality and the right panel on transverse momentum.

The ALICE data show less suppression compared to PHENIX results especially at low $p_{\mathrm{T}}$ [5]. At the LHC the charm quark density produced in the collisions increases with respect to RHIC energy. This may enhance the probability to create $\mathrm{J} / \psi$ from recombination of charm quarks. This contribution leads to an increase of the $R_{A A}$ at the LHC with respect to RHIC and from forward- to mid-rapidity, where the density of charm quarks is higher. Moreover, in order to recombine, two charm quarks need to be close enough in phase space, so the effect will be larger at low transverse momentum of $\mathrm{J} / \psi$. Comparison of the inclusive $\mathrm{J} / \psi$ production in $\mathrm{Pb}-\mathrm{Pb}$ collisions at $\sqrt{s_{\mathrm{NN}}}=5.02 \mathrm{TeV}$ with $\sqrt{s_{\mathrm{NN}}}=$ $2.76 \mathrm{TeV}$ data shows the same behavior and values in spite of the expected larger values of the charm quark density. However the theoretical calculations by statistic and dynamical models provide a good description of the measurements within uncertainties [6].

Inclusive $\mathrm{J} / \psi$ and $\psi(2 \mathrm{~S})$ productions have been studied at the LHC in $\mathrm{p}-\mathrm{Pb}$ collisions at energy $\sqrt{s_{\mathrm{NN}}}=5.02 \mathrm{TeV}$. The ALICE and LHCb results are in good agreement. At ALICE the measurement is performed in the center of mass rapidity domains $2.03<y<3.53$ and $-4.46<y<-2.96$ in muon channel and in electron channel in the center of mass rapidity domains $-1.37<y<0.43$ [13]. While at forward rapidity, corresponding to the proton direction, a suppression of the $\mathrm{J} / \psi$ yield with respect to binary-scaled pp collisions is observed, in the backward region no suppression is presented. Theoretical models including parton shadowing and coherent energy loss mechanism describe $\mathrm{J} / \psi$ production but could not reproduce additional suppression of $\psi(2 S)$ [14].

The inclusive production cross sections of $\Upsilon(1 S)$ and $\Upsilon(2 S)$ as a function of $p_{\mathrm{T}}$ and rapidity have been measured using ALICE detector at forward rapidity in pp collisions at $\sqrt{s_{\mathrm{NN}}}=7 \mathrm{TeV}$ [15]. The results are in good agreement with measurements from LHCb experiment [16] over similar $p_{\mathrm{T}}$ and rapidity ranges. They are complemented by CMS measurements at mid-rapidity [17]. They are also in good agreement with NRQCD calculations.

The ALICE measurement of inclusive $\Upsilon(1 \mathrm{~S})$ production in $\mathrm{Pb}-\mathrm{Pb}$ collisions was carried out at forward rapidity [18]. A strong suppression is observed with respect to pp collisions scaled by the number of independent nucleon-nucleon collisions. The ALICE results are compared with those of 
CMS $(|y|<2.4$. The observed suppression is stronger at forward than at mid-rapidity. The model [9] predicts a nearly constant $R_{A A}$ which is in disagreement with ALICE and CMS data.

The production of inclusive $\Upsilon(1 S)$ and $\Upsilon(2 S)$ was measured also in p-Pb collisions at $\sqrt{s_{\mathrm{NN}}}=$ $5.02 \mathrm{TeV}$ in ALICE. Theoretical models with partonic energy loss plus shadowing could describe the data at forward rapidity but underestimate the suppression at backward rapidity.

Quarkonium production at LHC is a useful probe for the investigation of QGP formation and for testing pQCD models in pp scattering. For $\mathrm{p}-\mathrm{Pb}$ collisions theoretical models including nuclear suppression, parton shadowing and coherent energy loss mechanisms could reproduce $\mathrm{J} / \psi$ production, but fail to describe additional suppression of $\psi(2 \mathrm{~S})$ and underestimate the observed $\Upsilon(1 \mathrm{~S})$ suppression at forward rapidity. In $\mathrm{Pb}-\mathrm{Pb}$ collisions the evidence for strong $\mathrm{J} / \psi$ suppression at large transverse momentum and additional $\mathrm{J} / \psi$ production from regeneration at low $p_{\mathrm{T}}$ was obtained. However, in order to get the ultimate data on the explanation of all observed effects in the anomalous charmonia states suppression due to the formation of Quark-Gluon Plasma we need to investigate the problem at intermediate energies.

\section{Experiments in fixed target mode at the LHC beams}

\subsection{Quarkonium production at fixed target}

Progress in our understanding of the properties of nuclear matter requires systematic and high statistical measurements also in low-energy region. The energy intervals between SPS, RHIC and LHC are very important to study the mechanism of quarkonium production and suppression, in order to investigate medium effects and conditions of quark-gluon plasma formation. Unfortunately, beam energy scan at RHIC produced data with low statistic and large systematic errors because the luminosity at RHIC decreases with energy reduction. It is important to study the mechanism of quarkonium production and suppression at low energies up to $35 \mathrm{GeV} /$ per nucleon at the FAIR in CBM and at NICA collider in Dubna with high statistics. At CERN SPS the fixed target experiment CHIC for charmonium study at energy up to $20 \mathrm{GeV}$ is under preparation. At RHIC there are experiments at fixed target at STAR installations. If the proton and ion beams would be used at the LHC with the fixed target, the energy interval between SPS energy $\left(\sqrt{s_{\mathrm{NN}}}=29 \mathrm{GeV}\right)$ and RHIC nominal energy $\left(\sqrt{s_{\mathrm{NN}}}=200 \mathrm{GeV}\right)$ in p-A and A-A collisions could be covered [19].

\subsection{Three approaches for the fixed target experiments at the LHC. The possibility to use polarized target}

There are three options of using high-intensity LHC beams for fixed target experiment AFTER (A Fixed Target ExpeRiment). The target in the form of thin ribbon could be placed around the main orbit of the LHC. The life time of the beam is determined by the beam-beam and beam-gas interactions. Therefore after some time the particles will leave the main orbit and interact with the target ribbon. So for fixed target measurements only halo of the beam will be used and the target can provide some extra collimation. Therefore no distortion of the main beam will be introduced. Since the target ribbon should not interfere during the beam formation and acceleration process it should be lifted in the working position after the tuning of the beam $[19,20]$. This type of experiment could be carried out at the existing installations (such as ALICE or LHCb). The experiment with the target in the form of thin ribbon placed in the beam halo may be the first stage of the multi-purpose experiment AFTER [21].

The second option is the installation of the internal gas target like the LHCb SMOG or HERMES systems. The internal gas system SMOG with low gas density is currently used by LHCb Collaboration as a luminosity monitor and was initially designed for the monitoring the transverse size of the 
beam. It works during extended period of time without any influence on the other LHC experiments. In 2012 and $2013 \mathrm{LHCb}$ measured p-Ne and Pb-Ne collisions with the c.m. NN energy $\sqrt{s_{\mathrm{NN}}}=$ $86.6 \mathrm{GeV}$ for proton beam and $54.5 \mathrm{GeV}$ for lead beam. During 2015 year the measuring of p-Ne, p-He and p-Ar collisions at $\sqrt{s_{\mathrm{NN}}}=110.4 \mathrm{GeV}$ and $\mathrm{Pb}$-Ar collisions at $\sqrt{s_{\mathrm{NN}}}=68.6 \mathrm{GeV}$ were successfully performed. No distortion of the LHC performance was observed. Using the system like the HERMES experiment at HERA the higher gas pressure could be obtained with the possibility of the gas target polarization [22].

The third option consists of using the bent crystal placed in the halo of the LHC beams. Several protons or lead ions in the bunch could be channeled along the crystal curvature. The extracted beam can be used with an extra beamline and special installation for polarization of the target. This extraction allows to obtain very clean and collimated beam. This technique does not affect the LHC performance as it will use only halo of the beam. Similar technique is investigated and currently used by the UA9 Collaboration as a possibility to improve the LHC beam collimation. In the case of the gas target the polarization is possible. Using the target similar to HERMES system, the effective polarization of $\mathrm{H}$ or $\mathrm{D}$ can reach 0.8 . Using extracted beam, different systems for target polarization could be used. In general, H or D gas targets provide good possibility for high value of polarization with similar data taking periods. The effective gas pressure could be similar to the HERMES and polarized target would have a length near one meter [22].

\subsection{The proposed measurements}

The experiment AFTER has a wide physical program, gives possibility to use different beams and targets of large thickness including the polarized ones. The physical program includes quarkonium production, the study of the Drell-Yan process, investigation of D-meson production, the study of flow, spin physics, energy scan for the search of the critical point of phase transition of nuclear matter to Quark-Gluon Plasma. Quarkonia are the important probes for investigation the deconfinement at ultrarelativistic heavy-ion collisions. But the mechanism of quarkonium production and suppression is not well understood in some energy and kinematical domain, especially at large Feynman $x_{\mathrm{F}}$, where cold nuclear matter effects expected to be large. The high statistical data in these domains give possibility to understand the mechanism of production and to estimate the contribution of recombination process. It would be possible to investigate production not only $\mathrm{J} / \psi, \psi(2 \mathrm{~S})$ and $\Upsilon(\mathrm{nS})$ mesons, but also $\eta_{c}$ and $\chi_{c}$ states. These measurements require high statistics, large acceptance and good efficiency. Investigation of Drell-Yan process is very promising task in itself, but it is also background process in quarkonium production. The study of $\mathrm{D}$-meson production is an important part of the measurement of charmonium production and dissociation.

Now RHIC is the only one collider of polarized protons with possibility to study spin physics at ultra-relativistic energies. There are no polarized beams at the Fermilab Tevatron or at the LHC. The polarized beams are planned to be build at the NICA collider in Dubna at low energy. But it becomes possible to perform new investigations of single transverse spin asymmetries (STSA), $A_{N}$, using a polarized fixed target at the LHC beams. It is connected with the Sivers effect [23] observed many years ago and provided the correlation of the transverse momentum of the partons, $k_{N}$ to the nucleon spin. This effect depends on transverse momentum distribution (TMD). In the case of fixed target experiment AFTER at the LHC beams because of the boost between laboratory and centre-of-mass systems, measurements of the STSAs in the rapidity range of experiments ALICE or LHCb would investigate the region of large parton momentum fraction in the polarized hadron, $x^{\uparrow}$ [21]. This is the range, where asymmetries are expected to be the largest. In the case of Drell-Yan, the quark Sivers function is predicted to have an opposite sign to the Deep Inelastic Scattering (DIS). It was investigated in the study of the Drell-Yan process in COMPASS at CERN and in Fermilab experiments 
with polarized targets at low values of $x^{\uparrow}$. The experiment AFTER could provide measurement of STSAs of Drell-Yan over a wide range of $x^{\uparrow}$ in a single experiment.

Practically nothing is known about the gluon Sivers effect. The PHENIX collaboration was the first to investigate STSAs of gluon sensitive probes in $\mathrm{J} / \psi$ production. The value of $A_{N}$ was found to be compatible with zero but with high errors, allowing the values as large as $20 \%$. In the experiment AFTER provided in the high luminosity at the LHC beams it would be possible to measure STSAs for bottomonia, spin-singlet states as $\eta_{c}$, to investigate gluon Sivers effect and importance of the gluon orbital angular momentum to solve the nucleon-spin problem.

\section{Conclusions}

The collisions of the high-energy LHC beams with fixed target, including polarized nuclei targets, can expand the range of fundamental physical investigations accessible at CERN. Using the proton and ion beams at the LHC with fixed targets, the data in the energy interval between maximum energy of the SPS and the nominal RHIC energy in p-A and A-A collisions could be obtained. The fixed target mode opens possibility for precise and unique measurements and advances our understanding in the fields of heavy-ion, hadron, spin and astroparticle physics, study of flow, energy scan for searching the critical point of the phase transition of matter to Quark-Gluon Plasma (QGP).

\section{References}

[1] T. Matsui and H. Satz, Phys. Lett. B 178, 416 (1986)

[2] S. Chatrchyan et al., CMS Collaboration, Phys. Rev. Lett. 109, 222301 (2012)

[3] S. Chatrchyan et al., CMS Collaboration, Phys. Rev. Lett. 107, 052302 (2011)

[4] B. Abelev et al., ALICE collaboration, Phys. Rev. Lett. 109, 072301 (2012)

[5] B. Abelev et al., ALICE collaboration, Phys. Lett. B 743, 314 (2014)

[6] J. Adams et al., ALICE collaboration, arXiv:1606.08197 [nucl-ex]

[7] R. L. Thews et al., Eur. Phys. J C 43, 97 (2005). R. L. Thews and M.I. Mangano, Phys. Rev. C 73, 014904 (2006)

[8] A. Andronic, P. Braun-Munzinger, K. Redlich, and J. Stachel, Phys. Rev. Lett. 659, 149 (2008)

[9] A. Emerick, X. Zhao and R. Rapp, Eur. Phys. J A 48, 72 (2012)

[10] H. Fujii, K. Watanabe, Nucl. Phys. A 915, 1 (2013)

[11] T. Dahms, CMS Collaboraion, J. Phys. G 38, 124105 (2011)

[12] A. Adare et al., PHENIX Collaboration, Phys. Rev. C 84, 054912 (2011)

[13] B. Abelev et al., ALICE collaboration, JHEP 02, 073 (2014)

[14] B. Abelev et al., ALICE collaboration, JHEP 12, 073 (2014)

[15] B. Abelev et al., ALICE collaboration, Eur. Phys. J C 73, 2662 (2013)

[16] R. Aaij et al., LHCb collaboration, Eur. Phys. J C 72, 2100 (2012)

[17] S. Chatrchyan et al., CMS Collaboration, Phys. Lett. B 727, 101 (2013)

[18] B. Abelev et al., ALICE collaboration, Phys. Rev. Lett. B 738, 361 (2014)

[19] A. B. Kurepin, N. S. Topilskaya and M. B. Golubeva, Phys. Atom. Nucl. 74, 446 (2011)

[20] A. B. Kurepin and N. S. Topilskaya, Adv. High Energy Phys. 2015, 760840 (2015)

[21] S. J.Brodsky, F. Fleuret, C. Hadjidakis, J. P. Lansberg, Phys. Rept. 522, 239 (2013)

[22] J. P. Lansberg et al., PoS (PSTP2015) 042 (2015)

[23] D. W. Sivers, Phys. Rev. D 41, 83 (1990) 